\title{
Prevalence, predictors, and prognostic implications of PR interval prolongation in patients with heart failure
}

\author{
Theodora Nikolaidou ${ }^{1}$ (D) Pierpaolo Pellicori ${ }^{1} \cdot$ Jufen Zhang $^{1,2} \cdot$ Syed Kazmi $^{1}$ \\ Kevin M. Goode ${ }^{1} \cdot J^{\prime}$ hn G. Cleland ${ }^{1,3} \cdot$ Andrew L. Clark ${ }^{1}$
}

Received: 19 April 2017 / Accepted: 13 September 2017 / Published online: 15 September 2017

(c) The Author(s) 2017. This article is an open access publication

\begin{abstract}
Aims To determine the prevalence, incidence, predictors, and prognostic implications of PR interval prolongation in patients referred with suspected heart failure.

Methods and results Consecutive patients referred with suspected heart failure were prospectively enrolled. After excluding patients with implantable cardiac devices and atrial fibrillation, 1420 patients with heart failure and reduced ejection fraction (HeFREF) [age: median 71 (interquartile range IQR 63-78) years; men: 71\%; NT-ProBNP: 1319 (583-3378) ng/L], 1094 with heart failure and normal ejection fraction (HeFNEF) [age: 76 (70-82) years; men: 47\%; NT-ProBNP: 547 (321-1171) ng/L], and 1150 without heart failure [age: 68 (60-75) years; men: 51\%; NTProBNP: 86 (46-140) ng/L] were included. The prevalence of first-degree heart block [heart rate corrected PR interval $(\mathrm{PRc})>200 \mathrm{~ms}$ ] was higher in patients with heart failure (21\% HeFREF, 20\% HeFNEF, 9\% without heart failure). In patients with HeFREF or HeFNEF, longer baseline PRc
\end{abstract}

This work was performed at the Department of Academic Cardiology, Castle Hill Hospital, University of Hull.

Electronic supplementary material The online version of this article (doi:10.1007/s00392-017-1162-6) contains supplementary material, which is available to authorized users.

Theodora Nikolaidou

t.nikolaidou@hull.ac.uk

1 Department of Academic Cardiology, Castle Hill Hospital, University of Hull, Level 1 Daisy Building, Castle Road, Hull HU16 5JQ, UK

2 Anglia Ruskin University, Cambridge, UK

3 National Heart and Lung Institute, Imperial College London and Robertson Centre for Biostatistics and Clinical Trials, University of Glasgow, Glasgow, UK was associated with greater age, male sex, and longer QRS duration, and, in those with HeFREF, treatment with amiodarone or digoxin. Patients with heart failure in the longest PRc quartile had worse survival compared to shorter PRc quartiles, but PRc was not independently associated with survival in multivariable analysis. For patients without heart failure, shorter baseline PRc was independently associated with worse survival.

Conclusion PRc prolongation is common in patients with HeFREF or HeFNEF and associated with worse survival, although not an independent predictor of outcome. The results of clinical trials investigating the therapeutic potential of shortening the PR interval by pacing are awaited.

Keywords First-degree heart block · Heart failure $\cdot$ PR interval
Abbreviations
CRT Cardiac resynchronization therapy
eGFR Estimated glomerular filtraion rate
HeFNEF Heart failure with normal ejection fraction
HeFREF Heart failure with reduced ejection fraction
LBBB Left bundle branch block
LVEF Left ventricular ejection fraction
NT-ProBNP Amino-terminal pro-B-type natriuretic peptide
NYHA New York Heart Association
TSH Thyroid stimulating hormone

\section{Introduction}

Abnormal myocardial function is often accompanied by disturbances in electrical conduction. Patients with heart failure with reduced ejection fraction (HeFREF) have a substantial 
prevalence and annual incidence of QRS prolongation [1, $2]$, both of which are associated with worse outcomes [2, 3]. However, there are few reports on the prevalence, incidence, and prognostic significance of the PR interval in ambulatory patients with chronic heart failure [4-6]. Analyses from studies in the general population have shown that PR interval prolongation is associated with increased risk of atrial fibrillation, pacemaker implantation, and mortality [7-10].

Most available data in heart failure relate to patients undergoing device implantation rather than coming from an unselected population [7]. Kronborg et al. reported that in 659 patients undergoing CRT implantation, $47 \%$ had firstdegree heart block, and that a long native PR interval was an independent predictor of all-cause and cardiac mortality [11]. In CARE-HF, a longer native PR interval at baseline and longer PR interval at 3 months (paced PR for the CRT group and native PR for the control group) predicted both all-cause mortality and urgent hospitalisation for heart failure even after adjusting for CRT [5]. In patients with advanced heart failure, up to half of arrhythmic deaths may be bradycardic in origin, including high degree atrioventricular block [12-14].

The introduction of cardiac resynchronisation therapy (CRT) has focussed interest on QRS duration and morphology in patients with heart failure. Although CRT was originally conceived by many as a treatment for ventricular dyssynchrony, imaging studies have cast doubt on this hypothesis, leaving considerable doubt about precisely how CRT exerts benefit. Indeed, it may be that the predominant mechanism of benefit of CRT varies from one patient to the next and over time, and might include reduction of atrioventricular delay. Recent reports $[6,15,16]$ suggest that PR prolongation, a potential surrogate for greater atrioventricular delay, might also be a target for CRT.

The aim of the present analysis was to describe the prevalence, incidence, predictors, and prognostic implications of a prolonged PR interval in patients with suspected heart failure and, if confirmed, with or without a reduced left ventricular ejection fraction (LVEF).

\section{Methods}

\section{Ethics approval}

The investigation conforms with the principles outlined in the Declaration of Helsinki. It was approved by the Hull and East Yorkshire Research Ethics Committee (Heart Care Study ELSY 2642). All subjects gave written informed consent.

\section{Participants}

Consecutive patients referred with suspected heart failure to a community heart failure clinic between 2001 and 2014 were enrolled. Only patients in sinus rhythm on their baseline electrocardiogram who did not have a ventricular pacing device were included in the analysis. Heart failure with reduced ejection fraction (HeFREF) was defined as the presence of symptoms compatible with the diagnosis of heart failure and impaired left ventricular (LV) systolic function measured as an LVEF $<45 \%$ on echocardiography where possible and estimated visually when not. Heart failure with normal ejection fraction (HeFNEF) was defined as the presence of symptoms compatible with the diagnosis of heart failure with $L V E F \geq 45 \%$ and, for these patients in sinus rhythm, an amino-terminal pro-B-type natriuretic peptide (NT-ProBNP) $\geq 220 \mathrm{ng} / \mathrm{mL}$. Patients with an LVEF $\geq 45 \%$ and an NT-ProBNP $<220 \mathrm{ng} / \mathrm{mL}$ were considered not to have heart failure.

Electrocardiographic intervals were obtained from automated analysis of the surface electrocardiogram (ECG). The only exclusion criteria were the inability to provide informed consent, pregnancy, atrial fibrillation, and an implanted cardiac device, even if not pacing at the time of the ECG recording, because the effect that pacing has on native ECG intervals is uncertain. For patients who received a cardiac device during follow-up, survival curves were censored at the time of implant (i.e., the patient was treated as lost to follow-up thereafter). Patients with paroxysmal atrial fibrillation who were in sinus rhythm on the baseline electrocardiogram were included in the analysis.

\section{Clinical assessment}

Baseline characteristics included medical history, therapy, height, weight and blood pressure, blood tests [standard haematology and biochemistry tests, thyroid stimulating hormone (TSH), and NT-ProBNP], and echo- and electrocardiographic data. Echocardiographic information included LVEF and the presence and severity of mitral regurgitation. Patients with diabetes included those managed by diet-alone or with insulin or other hypoglycaemic agents. Patients with the previous myocardial infarction, coronary artery bypass surgery, or positive tests for ischaemia were considered to have ischaemic heart disease (IHD). Cerebrovascular disease was defined as patients with a history of ischaemic stroke or transient ischaemic attack. The estimated glomerular filtraion rate (eGFR) was calculated using the abbreviated modification of the diet in renal disease (MDRD) equation [17]. 


\section{Definition of PRc}

Automated 12-lead ECG interval measurements were used to measure heart rate, PR, QRS, and QT intervals. PR interval reported was the longest measured PR interval in any lead. The PR interval duration changes with heart rate (longer PR interval at faster heart rates). Heart rate changes continually, therefore, to compare PR intervals taken at different heart rates, an adjustment is made (PRc) using a previously published formula [18]. We used PRc throughout our analysis, except in the hazard ratio analysis, where baseline heart rate is independently associated with worse survival in some groups, and for this reason, we present heart rate and unadjusted PR as separate variables. QT interval was also corrected for heart rate using Bazett's formula [19]. Firstdegree heart block was defined as PRc interval > $200 \mathrm{~ms}$.

\section{Follow-up}

Follow-up for all patients was censored at the last point of contact in primary or secondary care to avoid unreported deaths due, for instance, to emigration out of the region. Patients diagnosed with HeFREF were routinely given follow-up appointments at 4 and 12 months with other visits as clinically indicated. Patients in whom the diagnosis of heart failure was excluded, as well as many patients with HeFNEF, were discharged and followed up only through electronic primary and secondary care health records which usually did not include electrocardiographic data. Accordingly, electrocardiographic data during follow-up were only available for the patients with HeFREF.

\section{Statistical analysis}

Data are presented as median and interquartile range (IQR). Categorical data are presented as numbers and percentages. Differences between two independent groups of continuous data were tested using the independent $\underline{t}$ test. The Chi-square test was used for comparisons between independent groups of categorical data and a one-way ANOVA was used to compare more than two independent groups of continuous data. For paired samples, the paired $t$ test was used for continuous data. For categorical data with two or more categories, the McNemar's test and the marginal homogeneity non-parametric test were used, respectively. For comparisons across PRc quartiles, trend statistical tests were used. SPSS (version 22) statistical computer package was used for data analysis.

The distribution of NT-ProBNP and TSH was skewed; we, therefore, used non-parametric tests to compare groups of continuous data and log-transformed NT-ProBNP and TSH data to satisfy the assumptions of the models.

Pearson correlation coefficients were used to observe the correlation between baseline variables in patients with
HeFREF. Multiple linear regressions were used to identify variables associated with PRc interval and the Cox regression model was used to identify the variables associated with all-cause mortality. Only the significant variables identified using univariable analysis were used in the multivariable Cox regression models. We used two-tailed tests at a significance level of 0.05 .

\section{Results}

Of 2333 patients with HeFREF, 1950 with HeFNEF, and 1193 without heart failure, respectively, 913 (39\%), 856 (44\%), and 43 (4\%) were excluded, mainly because of atrial fibrillation or an implanted device (Fig. 1). This left 1,420 patients with HeFREF, 1094 with HeFNEF, and 1150 without heart failure for the analysis (Table 1). Patients with HeFREF were younger, more likely to be men and had lower body surface area compared to those with HeFNEF. Patients without heart failure were slightly younger than in the other two groups. On average, compared to those with HeFNEF, patients with HeFREF had a faster heart rate, longer PRc, QRS, and QTc intervals and lower systolic and diastolic blood pressures, but eGFR and plasma TSH and mortality at 12 months were similar.

Amongst patients with HeFREF, 21\% had a PRc $>200 \mathrm{~ms}$ compared to $20 \%$ of patients with HeFNEF $(P=0.33)$ and 9\% of those without heart failure $(P<0.001)$ (Supporting Fig. 1A), but the proportions with a PRc $>230 \mathrm{~ms}$ were substantially lower, 5,7 , and $2 \%$, respectively. With increasing quartile of PRc duration, in each diagnostic group, a higher proportion of patients were aged $>65$ years and men and median QRS duration increased (Table 2 and Supporting Tables 1,2). Patients with HeFREF and longer PRc were also in a worse New York Heart Association class, which had worse left ventricular systolic function and greater use of loop diuretics (Table 2). Patients with HeFNEF and longer PRc had greater use of loop diuretics (Supporting Table 1).

\section{Variables associated with a long PR interval}

In all diagnostic groups, heart rate was inversely related to QT, PR, and QRS intervals (Supporting Table 3). PRc correlated weakly with QRS $\left(R^{2}=0.04 ; P<0.001\right)$ and to a lesser extent with QTc duration $\left(R^{2}=0.005 ; P=0.01\right)$ but not heart rate (Supporting Table 3).

In patients without heart failure, longer PRc was positively correlated with a history of paroxysmal atrial fibrillation (1\% had paroxysmal atrial fibrillation in the lowest PRc quartile compared to 5\% in the highest PRc quartile; $P=0.03$; data not shown). This association was not present in patients with heart failure. 


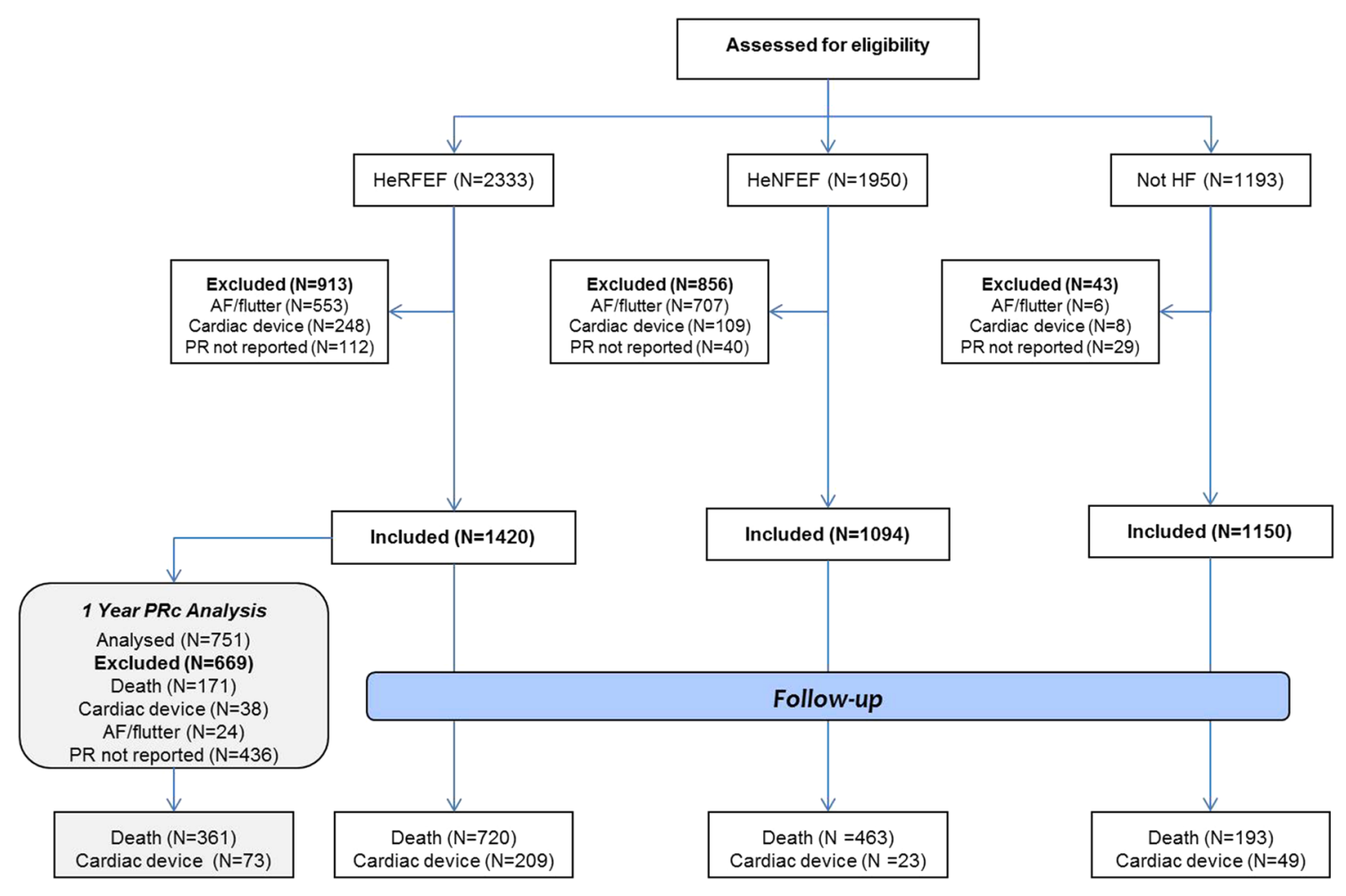

Fig. 1 Patient eligibility flow diagram

In a multivariable linear regression model, in patients with HeFREF, longer PRc interval was associated with greater age and male sex, longer QRS duration, and prescription of digoxin or amiodarone (Table 3). In patients with HeFNEF, longer PRc interval was associated with greater age and male sex, longer QRS duration, and prescription of $\beta$-blockers (Table 3). In patients without heart failure, longer PRc interval was associated with greater age and diabetes, longer QRS duration, lower eGFR, and prescription of $\beta$-blockers.

\section{One-year follow-up in patients with HeFREF}

Paired assessments at baseline and 1 year were available for 751 patients with HeFREF (Supporting Table 4); 171 had died, 24 developed atrial fibrillation, 38 had an implanted device, and 436 did not have a repeat electrocardiogram. Twenty-seven patients who developed AF at 1 year had an average baseline PRc of $179 \mathrm{~ms}$, while the 38 patients requiring implantation of any cardiac device had an average PRc of $184 \mathrm{~ms}$.

After 1 year, more patients were on heart failure medication compared to baseline, and LVEF had risen, New York Heart Association class and LV function had improved, NT-ProBNP had declined, and systolic and diastolic blood pressures had fallen. There was a decline in eGFR. Median PRc interval increased slightly further over 1 year $[173 \mathrm{~ms}$
(156-193) to $176 \mathrm{~ms}(158-197) ; P<0.001$; Supporting Table 4]. The prevalence of first-degree heart block was similar at baseline $(17 \%)$ and 1 year $(20 \% ; P=0.33) ; 39$ patients with first-degree heart block at baseline had normal values by 1 year, while 52 patients developed new first-degree heart block. PRc duration increased by $\geq 5 \%$ in $262(35 \%)$ patients with HeFREF, while it decreased by $\geq 5 \%$ in 161 (21\%) (Supporting Fig. 1B-C). Change by more than one quartile was rare (Supporting Fig. 1C). Only patients with a documented ECG at 1 year were included in this analysis. This may have resulted in selection bias.

\section{Survival in patients with HeFREF}

During a median follow-up of 3.9 years (IQR: 1.6-7.1), with follow-up censored at the time of death, 627 (44\%; 11\% per annum) patients died (Fig. 2a). Patients in the highest baseline PRc quartile had a worse survival compared to all other quartiles. In a Cox regression model, baseline variables independently associated with worse survival were: increasing $\log$ [NT-ProBNP], age, and NYHA class; and decreasing diastolic blood pressure and eGFR and the use of digoxin but neither PR nor PRc (Table 4).

Survival was worse in those with first-degree heart block in the second and subsequent years of follow-up especially if PRc increased between baseline and the first year (Supporting Fig. 2). $\beta$-blockers were started in 209 patients between 
Table 1 Baseline demographic and clinical characteristics

\begin{tabular}{|c|c|c|c|c|c|c|c|c|}
\hline & $\begin{array}{l}\text { Missing } \\
N\end{array}$ & $\begin{array}{l}\text { HeFREF } \\
(N=1420)\end{array}$ & $\begin{array}{l}\text { HeFNEF } \\
(N=1094)\end{array}$ & $\begin{array}{l}\text { Not HF } \\
(N=1150)\end{array}$ & $\begin{array}{l}P \text { value } \\
\text { HeFREF } \\
\text { vs. Not } \\
\text { HF }\end{array}$ & $\begin{array}{l}P \text { value } \\
\text { HeFREF } \\
\text { vs. HeF- } \\
\text { NEF }\end{array}$ & $\begin{array}{l}P \text { value } \\
\text { HeFNEF } \\
\text { vs. Not } \\
\text { HF }\end{array}$ & $\begin{array}{l}P \text { value } \\
\text { All groups }\end{array}$ \\
\hline Age (years) & - & $71(63-78)$ & $76(70-82)$ & $68(60-75)$ & $<0.001$ & $<0.001$ & $<0.001$ & $<0.001$ \\
\hline Men $(\%)$ & - & $1005(71)$ & $516(47)$ & $584(51)$ & $<0.001$ & $<0.001$ & 0.156 & $<0.001$ \\
\hline NYHA class (\%) & $2 / 17 / 161$ & & & & & & & \\
\hline I & & $212(15)$ & $325(30)$ & 488 (49) & $<0.001$ & $<0.001$ & $<0.001$ & $<0.001$ \\
\hline II & & $708(50)$ & $493(46)$ & 369 (37) & & & & \\
\hline III & & $457(32)$ & $244(23)$ & $124(13)$ & & & & \\
\hline IV & & $41(3)$ & $15(1)$ & $8(1)$ & & & & \\
\hline Diabetes (\%) & $2 / 3 / 4$ & $356(25)$ & $287(26)$ & $259(23)$ & 0.12 & 0.36 & 0.06 & 0.13 \\
\hline $\begin{array}{l}\text { Ischaemic heart disease } \\
(\%)\end{array}$ & $2 / 7 / 15$ & $932(66)$ & $476(44)$ & $244(22)$ & $<0.001$ & $<0.001$ & $<0.001$ & $<0.001$ \\
\hline $\begin{array}{l}\text { Cerebrovascular dis- } \\
\text { ease }(\%)\end{array}$ & $2 / 7 / 15$ & $100(7)$ & $68(6)$ & $40(4)$ & $<0.001$ & $<0.001$ & 0.01 & 0.001 \\
\hline Body surface area $\left(\mathrm{m}^{2}\right)$ & $6 / 7 / 4$ & $1.91(1.73-2.08)$ & $1.85(1.71-2.04)$ & $1.95(1.78-2.11)$ & $<0.001$ & 0.01 & $<0.001$ & $<0.001$ \\
\hline Systolic BP (mmHg) & $13 / 3 / 21$ & $129(114-147)$ & $149(131-167)$ & $147(132-162)$ & $<0.001$ & $<0.001$ & 0.06 & $<0.001$ \\
\hline Diastolic BP (mmHg) & $13 / 3 / 21$ & $76(66-84)$ & $78(70-88)$ & $83(75-91)$ & $<0.001$ & $<0.001$ & $<0.001$ & $<0.001$ \\
\hline Heart rate (bpm) & - & $73(63-86)$ & $68(59-78)$ & $69(60-79)$ & $<0.001$ & $<0.001$ & 0.18 & $<0.001$ \\
\hline QRS (ms) & $6 / / 1 / 4$ & $112(96-140)$ & $92(84-106)$ & $90(82-98)$ & $<0.001$ & $<0.001$ & $<0.001$ & $<0.001$ \\
\hline $\mathrm{QRS} \geq 150 \mathrm{~ms}(\%)$ & $6 / / 1 / 4$ & $249(18)$ & $51(5)$ & $18(2)$ & $<0.001$ & $<0.001$ & $<0.001$ & $<0.001$ \\
\hline $\mathrm{PR}(\mathrm{ms})$ & - & $172(154-194)$ & $168(152-192)$ & $162(148-180)$ & $<0.001$ & 0.53 & $<0.001$ & $<0.001$ \\
\hline PRc (ms) & - & $174(157-196)$ & $168(151-192)$ & $163(147-179)$ & $<0.001$ & 0.03 & $<0.001$ & $<0.001$ \\
\hline QT (ms) & $19 / 19 / 6$ & $413(380-446)$ & $406(384-431)$ & $396(372-416)$ & $<0.001$ & $<0.001$ & $<0.001$ & $<0.001$ \\
\hline QTc (ms) & $19 / 19 / 6$ & $453(422-483)$ & $429(410-452)$ & $418(401-441)$ & $<0.001$ & $<0.001$ & $<0.001$ & $<0.001$ \\
\hline TSH (mIU/L) & $117 / 91 / 196$ & $1.6(1.0-2.5)$ & $1.7(1.1-2.5)$ & $1.6(1.0-2.3)$ & 0.04 & 0.37 & 0.01 & 0.02 \\
\hline $\begin{array}{l}\text { eGFR }(1.73 \mathrm{~mL} / \mathrm{min} / \\
\left.\mathrm{m}^{2}\right)\end{array}$ & $3 / 2 / 12$ & $62(47-77)$ & $61(47-75)$ & $75(63-88)$ & $<0.001$ & 0.15 & $<0.001$ & $<0.001$ \\
\hline NT-ProBNP (ng/L) & 436/8/139 & $1319(583-3378)$ & $547(321-1171)$ & $86(46-140)$ & $<0.001$ & $<0.001$ & $<0.001$ & $<0.001$ \\
\hline $\begin{array}{l}\text { Ejection fraction by } \\
\text { Simpson's }\end{array}$ & $564 / 523 / 491$ & $33(27-37)$ & $54(48-61)$ & $59(54-64)$ & $<0.001$ & $<0.001$ & $<0.001$ & $<0.001$ \\
\hline $\begin{array}{l}\text { LV systolic dysfunc- } \\
\text { tion }(\%)\end{array}$ & - & & & & & & & \\
\hline Normal-trivial & & 0 & $820(75)$ & $1151(100)$ & $<0.001$ & $<0.001$ & $<0.001$ & $<0.001$ \\
\hline Mild & & 0 & $274(25)$ & 0 & & & & \\
\hline Mild-moderate & & $849(60)$ & 0 & 0 & & & & \\
\hline$>$ Moderate & & $571(40)$ & 0 & 0 & & & & \\
\hline $\begin{array}{r}\text { Mitral regurgita- } \\
\text { tion }>\text { mild }(\%)\end{array}$ & 27/17/161 & 447 (32) & $124(13)$ & $27(2)$ & $<0.001$ & $<0.001$ & $<0.001$ & $<0.001$ \\
\hline \multicolumn{9}{|l|}{$\begin{array}{l}\text { Medication at initial } \\
\text { visit }\end{array}$} \\
\hline$\beta$-blocker (\%) & $14 / 18 / 55$ & $862(61)$ & $550(51)$ & $285(26)$ & $<0.001$ & $<0.001$ & $<0.001$ & $<0.001$ \\
\hline ACE-I (\%) & $14 / 18 / 55$ & $1019(72)$ & $532(49)$ & $325(30)$ & $<0.001$ & $<0.001$ & $<0.001$ & $<0.001$ \\
\hline $\mathrm{ARB}(\%)$ & $14 / 18 / 55$ & $133(9)$ & $161(15)$ & $128(12)$ & 0.07 & $<0.001$ & 0.02 & $<0.001$ \\
\hline MRA (\%) & $14 / 18 / 55$ & $422(30)$ & $95(9)$ & $22(2)$ & $<0.001$ & $<0.001$ & $<0.001$ & $<0.001$ \\
\hline Amiodarone (\%) & $6 / 18 / 36$ & $66(5)$ & $19(2)$ & $6(0.5)$ & $<0.001$ & 0.001 & $<0.001$ & $<0.001$ \\
\hline Digoxin no. (\%) & $6 / 18 / 36$ & $118(8)$ & $40(4)$ & $9(0.8)$ & $<0.001$ & $<0.001$ & $<0.001$ & $<0.001$ \\
\hline Loop Diuretic (\%) & $8 / 18 / 3$ & $993(70)$ & $557(52)$ & $249(22)$ & $<0.001$ & $<0.001$ & $<0.001$ & $<0.001$ \\
\hline Ivabradine (\%) & $14 / 18 / 55$ & $5(0.4)$ & $2(0.2)$ & 0 & 0.07 & 0.35 & 0.5 & 0.14 \\
\hline 1 year mortality (\%) & - & $171(12)$ & $76(10)$ & $8(1)$ & $<0.001$ & 0.13 & $<0.001$ & $<0.001$ \\
\hline
\end{tabular}


Table 1 (continued)

Bold indicates significance at the 0.05 level

Continuous variables are presented as median (interquartile range), whereas categorical variables are expressed as percentage. $P$ values are for differences between patients with HeFREF, HeFNEF, and those without heart failure. Pair-wise comparisons were performed using the independent $t$ test for continuous data (except for TSH and NT-ProBNP where the Mann-Whitney test was used) and the Chi-square test for categorical data. The one-way ANOVA test was used for comparisons of continuous data across all groups (except for TSH and NT-ProBNP where the Kruskal-Wallis test was used) and the Chi-square test for categorical data

$A C E-I$ angiotensin converting enzyme inhibitor, $A R B$ angiotensin receptor blocker, $B P$ blood pressure, $e G F R$ estimated glomerular filtration rate, $M R A$ mineralocorticoid receptor antagonist, NYHA New York Heart Association, TSH thyroid stimulating hormone

baseline and 1 year. Of these, 46 had first-degree heart block at baseline and a further $24(12 \%)$ developed it by 1 year. There was no interaction between commencing $\beta$-blockers, new first-degree heart block at 1 year and survival.

\section{Survival in patients with HeFNEF}

During a median follow-up of 3.7 years (IQR: 1.7-7.4), with follow-up censored at the time of death, 444 ( $41 \% ; ~ 11 \%$ per annum) patients died. Patients in the highest baseline PRc quartile had a worse survival compared to all other quartiles (Fig. 2b). In a Cox regression model, baseline variables independently associated with worse survival in HeFNEF were: increasing $\log [\mathrm{NT}-\mathrm{ProBNP}$, male sex, higher New York Heart Association class, and age. Neither PR nor PRc was independent predictors of outcome. The presence of diabetes, a lower body surface area, and the use of a loop diuretic or digoxin were also associated with worse survival (Table 4). A slower baseline heart rate was associated with better survival.

\section{Survival in patients without heart failure}

During a median follow-up of 7.9 years (IQR 3.2-11.2), only 190 (17\%; 2\% per annum) patients died. Follow-up was much longer than in the heart failure groups because of low mortality leading to low rates of censorship due to death. On an unadjusted analysis of quartiles, shorter PRc and a faster heart rate were independently associated with worse survival $(P=0.02$ each). Increasing $\log$ [NT-ProBNP], male sex and increasing age were associated with worse survival in patients without heart failure as was worse functional class (132 patients in NYHA III/IV) and the use of loop diuretics (249 patients). Of patients taking loop diuretics (249 patients), 73 died (29\%; $4 \%$ per annum). Of patients with an NT-ProBNP between 125 and $220 \mathrm{ng} / \mathrm{L}$ (310 patients), 80 died (26\%; 3\% per annum). Many patients had chronic lung disease accounting for symptoms.

\section{Device implantation}

During follow-up, 144 patents with HeFREF were implanted with CRT with or without a defibrillator. Twenty-nine patients were implanted with defibrillator alone, while 36 patients had a pacemaker. Thirty-six patients with HeFNEF required a pacemaker during follow-up as did 20 patients without heart failure. Increasing PR and QRS interval duration as well as advancing age were associated with a greater risk of pacemaker implantation (Supporting Table 5).

\section{Discussion}

This study is the first large epidemiological study to report the prevalence and predictors of a long PR interval in patients with heart failure. We found that modest increases in PR duration are common in heart failure with or without a reduced LVEF.

The prevalence of first-degree heart block in patients with HeFREF was $21 \%$. In the subgroup of patients with moderate-severe $\mathrm{LV}$ dysfunction and $\mathrm{QRS} \geq 130 \mathrm{~ms}(N=227)$, the prevalence on first-degree heart block was $29 \%$. This is consistent with the previous studies in patients with heart failure, $\mathrm{LVEF}<35 \%$, and left bundle branch block undergoing CRT, where the prevalence of first-degree heart block was reported to be between $26-52 \%[5,6,20]$. Amongst patients with $\mathrm{HeFNEF}$ and $\mathrm{QRS} \geq 130 \mathrm{~ms}(N=115)$, the prevalence of first-degree heart block was even higher (40\%).

A long PR interval in heart failure is likely to reflect widespread electrophysiological abnormalities, including atrial enlargement and myocardial fibrosis, atrioventricular nodal conduction delay and/or bundle branch/Purkinje fibre conduction delay, altered autonomic tone, and the effects of pharmacological interventions [21]. Studies of animal models of heart failure have shown anatomical and ion channel changes in the atria and atrioventricular node associated with delayed atrioventricular conduction [22].

PRc was not an independent predictor of outcome in patients with heart failure. This adds to existing evidence on first-degree heart block in other populations. Data from the Finish Social Insurance Institution's Coronary Heart Disease (CHD) Study showed no increased risk of mortality, hospitalisation, or incidence of atrial fibrillation, heart failure, or stroke with first-degree heart block in the general population [23]. In contrast, the Framingham study reported an increased risk of atrial fibrillation, pacemaker implantation, 
Table 2 Baseline demographic and clinical characteristics of patients with HeFREF, classified by PRc quartiles

\begin{tabular}{|c|c|c|c|c|c|}
\hline & PRc Q1 $N=355$ & PRc Q2 $N=355$ & PRc Q3 $N=355$ & PRc Q4 $N=355$ & $P$ value \\
\hline Age (years) & $70(61-77)$ & $70(61-77)$ & $71(63-78)$ & $73(65-79)$ & $<0.001$ \\
\hline Men, no. (\%) & $225(63)$ & $236(67)$ & $259(72)$ & $285(81)$ & $<0.001$ \\
\hline \multicolumn{6}{|l|}{ NYHA class, no. (\%) } \\
\hline I & $59(17)$ & $51(14)$ & $54(15)$ & $47(13)$ & 0.002 \\
\hline II & $193(54)$ & $180(51)$ & $171(48)$ & $164(46)$ & \\
\hline III & $96(27)$ & $114(32)$ & $122(35)$ & $125(36)$ & \\
\hline IV & $7(2)$ & $9(3)$ & $6(2)$ & $19(5)$ & \\
\hline Diabetes, no. (\%) & $64(18)$ & $83(23)$ & $96(27)$ & $113(32)$ & 0.001 \\
\hline Ischaemic heart disease, no. (\%) & $232(65)$ & $232(65)$ & $228(64)$ & $240(68)$ & 0.64 \\
\hline Cerebrovascular disease, no. (\%) & $17(5)$ & $30(8)$ & $28(8)$ & $28(8)$ & 0.19 \\
\hline Body surface area $\left(\mathrm{m}^{2}\right)$ & $1.86(1.67-2.05)$ & $1.90(1.71-2.08)$ & $1.91(1.75-2.07)$ & $1.95(1.78-2.11)$ & $<0.001$ \\
\hline Systolic BP (mmHg) & $130(115-147)$ & $129(115-147)$ & $130(115-147)$ & $128(112-147)$ & 0.69 \\
\hline Diastolic BP (mmHg) & $75(67-83)$ & $75(66-84)$ & $78(69-87)$ & $75(65-83)$ & 0.89 \\
\hline Heart rate (bpm) & $70(60-84)$ & $74(64-87)$ & $72(62-86)$ & $73(62-86)$ & 0.34 \\
\hline QRS (ms) & $104(92-134)$ & $106(96-136)$ & $114(98-142)$ & $120(104-148)$ & $<0.001$ \\
\hline $\mathrm{QRS} \geq 150 \mathrm{~ms}(\%)$ & $44(12)$ & $51(14)$ & $69(19)$ & $88(25)$ & $<0.001$ \\
\hline $\mathrm{PR}(\mathrm{ms})$ & $144(134-151)$ & $164(160-168)$ & $182(176-188)$ & $210(200-222)$ & - \\
\hline PRc (ms) & $146(136-152)$ & $166(162-170)$ & $184(178-189)$ & $212(203-224)$ & - \\
\hline QT (ms) & $417(382-446)$ & $406(374-440)$ & 412 (385-449) & $418(384-452)$ & 0.15 \\
\hline QTc (ms) & 447 (419-478) & $453(421-482)$ & $454(422-486)$ & $456(424-484)$ & 0.01 \\
\hline Thyroid stimulating hormone (mIU/L) & $1.5(1.0-2.4)$ & $1.5(1.0-2.3)$ & $1.7(1.0-2.6)$ & $1.7(1.1-2.7)$ & 0.01 \\
\hline eGFR $\left(1.73 \mathrm{~mL} / \mathrm{min} / \mathrm{m}^{2}\right)$ & $66(50-81)$ & $63(48-79)$ & $61(46-77)$ & $58(44-71)$ & $<0.001$ \\
\hline NT-ProBNP (ng/L) & $1311(582-3045)$ & $1048(446-3331)$ & $1400(548-3350)$ & $1751(811-3915)$ & 0.50 \\
\hline Ejection fraction by Simpson's & $32(27-38)$ & $33(27-38)$ & $33(27-37)$ & $32(25-37)$ & 0.19 \\
\hline Left ventricular dysfunction, no. (\%) & & & & & 0.02 \\
\hline Normal-trivial & - & - & - & - & \\
\hline Mild & 0 & 0 & 0 & 0 & \\
\hline Mild-moderate & $227(64)$ & $213(60)$ & $213(60)$ & $196(55)$ & \\
\hline$>$ Moderate & $128(36)$ & $142(40)$ & $142(40)$ & $159(45)$ & \\
\hline Mitral regurgitation $>$ mild & $108(31)$ & $100(29)$ & $124(36)$ & $115(33)$ & 0.23 \\
\hline$\beta$-blocker, no. $(\%)$ & $208(60)$ & $222(63)$ & $214(61)$ & $218(61)$ & 0.57 \\
\hline ACE-I, no. $(\%)$ & $259(74)$ & $254(72)$ & $251(71)$ & $255(73)$ & 0.72 \\
\hline ARB, no. (\%) & $31(9)$ & $37(10)$ & $26(7)$ & $39(11)$ & 0.59 \\
\hline MRA, no. (\%) & $87(25)$ & $109(31)$ & $115(32)$ & $111(32)$ & 0.05 \\
\hline Amiodarone, no. (\%) & $14(4)$ & $9(3)$ & $14(4)$ & $29(8)$ & 0.005 \\
\hline Digoxin, no. (\%) & $20(6)$ & $28(8)$ & $32(9)$ & $38(11)$ & 0.01 \\
\hline Loop diuretic, no. (\%) & $234(67)$ & $237(67)$ & $246(70)$ & $276(78)$ & 0.001 \\
\hline Ivabradine, no. (\%) & $3(0.8)$ & $1(0.3)$ & 0 & $1(0.3)$ & 0.16 \\
\hline 1 year mortality, no. (\%) & $36(10)$ & 49 (14) & $36(11)$ & 49 (13) & 0.34 \\
\hline
\end{tabular}

Bold indicates significance at the 0.05 level

Continuous variables are presented as median (interquartile range), whereas categorical variables are expressed as numbers (percentage). $P$ values are for differences between PRc quartiles (columns 2, 3, 4, and 5). The one-way ANOVA linear trend test was used for comparisons of continuous data across groups and the Cochran's Chi-square trend test for categorical data

$A C E-I$ angiotensin converting enzyme inhibitor, $A R B$ angiotensin receptor blocker, $B P$ blood pressure, $e G F R$ estimated glomerular filtration rate, $M R A$ mineralocorticoid receptor antagonist, NYHA New York Heart Association. See also Supporting Tables 1 and 2

and death with PR interval prolongation at 20 years' follow-up [8]. In patients with stable coronary artery disease and normal ejection fraction, first-degree heart block was associated with increased mortality and hospitalisation for heart failure in one study [24].

Patients with heart failure and first-degree heart block undergoing CRT have worse prognosis compared to those 


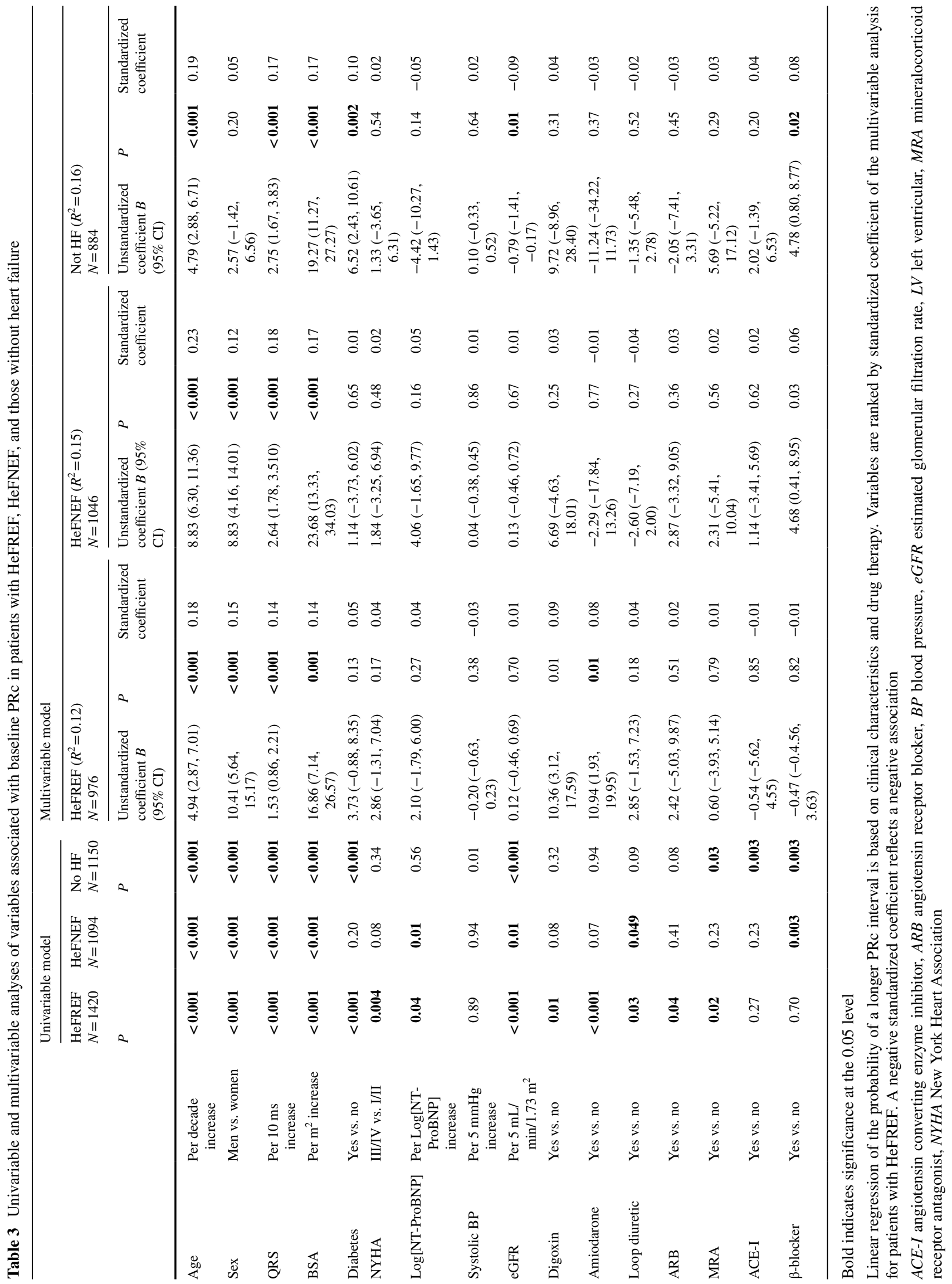




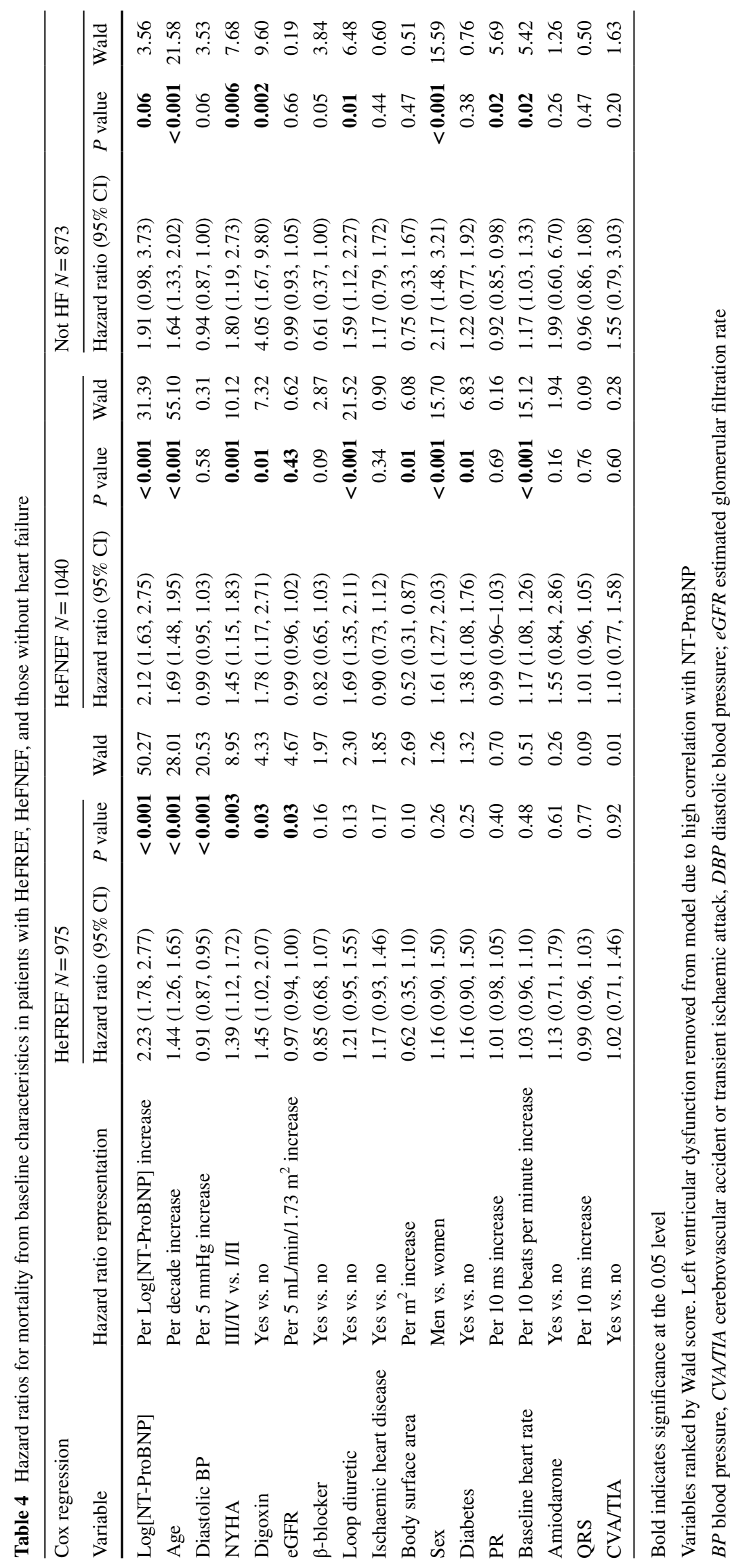



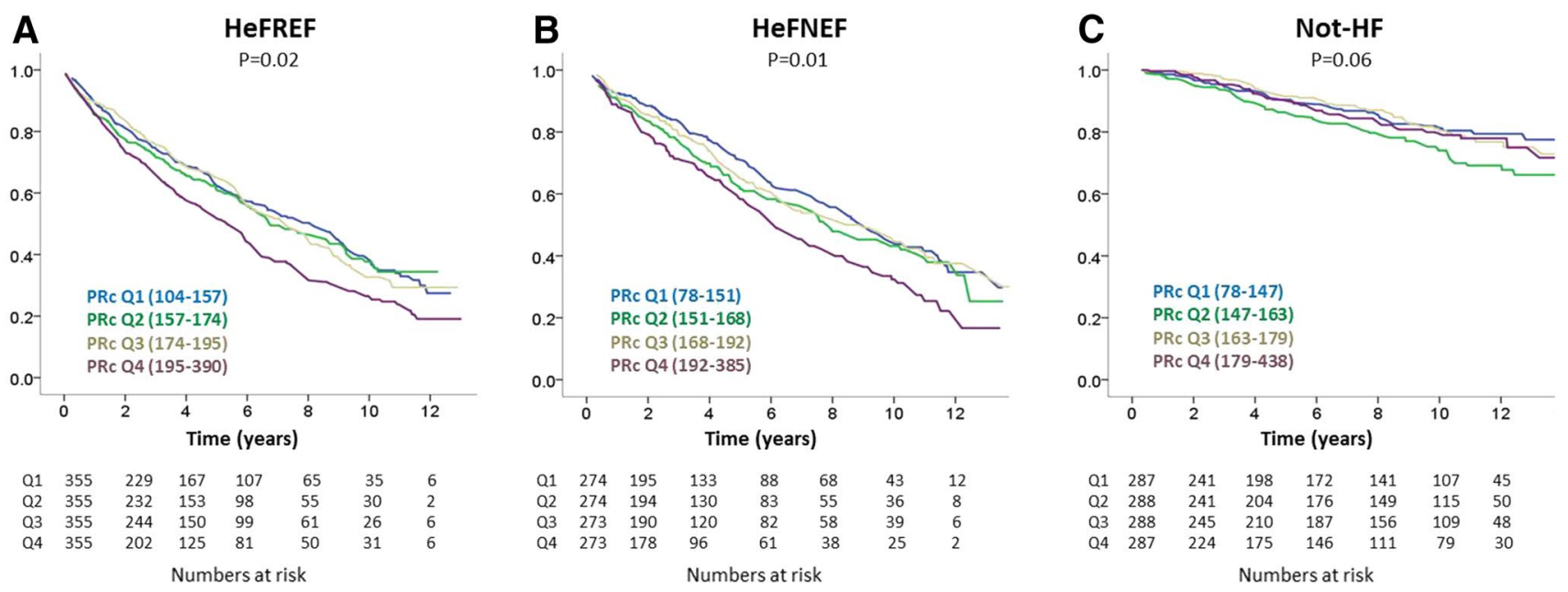

Fig. 2 Survival in patients with $\operatorname{HeFREF}(\mathbf{a}), \operatorname{HeFNEF}(\mathbf{b})$, and without heart failure (c) according to baseline PRc quartile (PRc ranges shown in brackets are in milliseconds)

with a normal PR interval [25, 26]. However, patients with first-degree heart block may derive greater benefit from CRT compared to pharmacological therapy [15]. In the COMPANION trial, a PR interval $>200 \mathrm{~ms}$ was associated with a $41 \%$ increased risk of the composite outcome of all-cause mortality or heart failure hospitalisation in those assigned to pharmacological therapy but was not higher amongst patients assigned to CRT [6]. A post hoc analysis from MADIT-CRT suggested that patients who did not have LBBB but did have a long PR interval benefited from CRT, whereas those with a normal PR interval did not [15]. The HOPE-HF trial is currently testing whether CRT with atrioventricular optimisation and His bundle pacing is beneficial in patients with heart failure and long PR interval without left bundle branch block [27].

In patients without heart failure, a short rather than a long PRc independently predicted increased mortality. This is in keeping with the previous findings in healthy population studies and in patients with coronary artery disease, suggesting that a short PR interval may not be a benign finding [28, 29]. It is unknown whether a short PR interval is a marker of underlying cardiac pathology (such as the presence of a concealed accessory pathway) or impaired autonomic/electrotonic regulation in the atrioventricular node.

Prolongation of QRS is common in patients with heart failure and is associated with worse outcomes [2, 3]. We found that in patients with heart failure (with or without reduced ejection fraction), neither QRS nor PRc interval duration was independently associated with all-cause mortality in models that included powerful prognostic markers such as age and NT-ProBNP. In the Korean heart failure registry $(N=1986), 16 \%$ of patients presenting with acute heart failure had first-degree atrioventricular block. The combination of first-degree atrioventricular block with a
QRS duration $\geq 120 \mathrm{~ms}$ was associated with an increased risk of adverse outcomes, including all-cause mortality [4]. In our study, patients with $P R c \geq 200 \mathrm{~ms}$ and QRS $\geq 130 \mathrm{~ms}$ had worse survival compared to other groups (Supporting Fig. 3), but combined PRc/QRS duration was not an independent predictor of mortality. Our study shows that similar to data from the general population [8], a long PR interval is associated with higher risk of simple pacemaker implantation in patients with heart failure who do not meet criteria for CRT or defibrillator.

$\beta$-blockade, amiodarone, and digoxin all prolong atrioventricular conduction. Patients on amiodarone are likely to have experienced prior atrial or ventricular arrhythmias, and may be more likely to have severe underlying heart disease and conduction abnormalities. However, $\beta$-blockade was not associated with prolongation of the PRc interval in patients with HeFREF; the atrioventricular node may be insensitive to $\beta$-blockade-induced conduction delay in heart failure, possibly due to down-regulation of adrenergic receptors or a reduction in parasympathetic tone.

A long PR interval is associated with an increased risk of subsequently developing atrial fibrillation in the general population [30]. Our data support this finding in patients without heart failure, in whom longer PRc was positively correlated with a history of paroxysmal atrial fibrillation (1\% in the first quartile of PRc had paroxysmal atrial fibrillation compared to $5 \%$ in the fourth quartile; $P=0.03$; data not shown).

\section{Study limitations}

We used a single electrocardiogram to measure PR interval. The PR interval varies during the day and under different physiological conditions. We did not have 24-h monitoring 
data. Patients with severe conducting disease were excluded, because they had an implanted cardiac device. The definition of first-degree heart block as $>200 \mathrm{~ms}$ is arbitrary and for this reason, PR interval duration was used as continuous variable in prognostic models. Due to the observational nature of this study, it is possible that unknown confounding factors may have affected our findings. We necessarily excluded patients with atrial fibrillation. At baseline, atrial fibrillation was common particularly in patients with normal ejection fraction, in whom the prevalence was $36 \%$ compared to $5 \%$ in patients with HeFREF. This finding is consistent with the previous reports [13] and suggests that atrial fibrillation might be a cause of HeFNEF. Patients with atrial fibrillation might have more severe atrioventricular conduction disease than patients in sinus rhythm, but it will be concealed. If so, we will have underestimated the true prevalence and consequences of conducting system disease in patients with heart failure, especially those with normal ejection fraction.

\section{Conclusions}

A prolonged PR interval is common in patients with chronic heart failure regardless of left ventricular ejection fraction. Although patients with heart failure and a longer PRc have worse survival, PRc is not independently associated with prognosis. Whether PR prolongation is a therapeutic target for pacing therapies in patients with heart failure is currently being tested in randomised trials.

\section{Compliance with ethical standards}

Funding This article/paper/report presents independent research funded by the National Institute for Health Research (NIHR). JGC is an NIHR Senior Investigator. TN is an NIHR Clinical Lecturer. The views expressed are those of the author(s) and not necessarily those of the NHS, the NIHR, or the Department of Health.

Conflict of interest JGC has received research grants, support and personal honoraria from Biotronik, Boston Scientific, LivaNova, Medtronic, and St Jude (not relating to the work under submission). $\mathrm{TN}$ has received research funding from St Jude (not relating to the work under submission). The remaining authors have no disclosures.

Open Access This article is distributed under the terms of the Creative Commons Attribution 4.0 International License (http://creativecommons.org/licenses/by/4.0/), which permits unrestricted use, distribution, and reproduction in any medium, provided you give appropriate credit to the original author(s) and the source, provide a link to the Creative Commons license, and indicate if changes were made.

\section{References}

1. Clark AL, Goode K, Cleland JG (2008) The prevalence and incidence of left bundle branch block in ambulant patients with chronic heart failure. Eur J Heart Fail 10:696-702

2. Sandhu R, Bahler RC (2004) Prevalence of QRS prolongation in a community hospital cohort of patients with heart failure and its relation to left ventricular systolic dysfunction. Am J Cardiol 93:244-246

3. Baldasseroni S, Opasich C, Gorini M, Lucci D, Marchionni N, Marini M, Campana C, Perini G, Deorsola A, Masotti G, Tavazzi L, Maggioni AP (2002) Left bundle-branch block is associated with increased 1-year sudden and total mortality rate in 5517 outpatients with congestive heart failure: a report from the Italian network on congestive heart failure. Am Heart J 143:398-405

4. Park SJ, On YK, Byeon K, Kim JS, Choi JO, Choi DJ, Ryu KH, Jeon ES (2013) Short- and long-term outcomes depending on electrical dyssynchrony markers in patients presenting with acute heart failure: clinical implication of the first-degree atrioventricular block and QRS prolongation from the Korean Heart Failure registry. Am Heart J 165:57-64

5. Gervais R, Leclercq C, Shankar A, Jacobs S, Eiskjaer H, Johannessen A, Freemantle N, Cleland JG, Tavazzi L, Daubert C (2009) Surface electrocardiogram to predict outcome in candidates for cardiac resynchronization therapy: a sub-analysis of the CARE-HF trial. Eur J Heart Fail 11:699-705

6. Olshansky B, Day JD, Sullivan RM, Yong $P$, Galle E, Steinberg JS (2012) Does cardiac resynchronization therapy provide unrecognized benefit in patients with prolonged PR intervals? The impact of restoring atrioventricular synchrony: an analysis from the COMPANION Trial. Heart Rhythm 9:34-39

7. Nikolaidou T, Ghosh J, Clark A (2016) Outcomes related to first degree atrioventricular block and therapeutic implications in patients with heart failure. JACC Clin Electrophysiol 2(2):181-92

8. Cheng S, Keyes MJ, Larson MG, McCabe EL, Newton-Cheh C, Levy D, Benjamin EJ, Vasan RS, Wang TJ (2009) Long-term outcomes in individuals with prolonged PR interval or first-degree atrioventricular block. JAMA 301(24):2571-2577

9. Kwok CS, Rashid M, Beynon R, Barker D, Patwala A, MorleyDavies A, Satchithananda D, Nolan J, Myint PK, Buchan I, Loke YK, Mamas MA (2016) Prolonged PR interval, first-degree heart block and adverse cardiovascular outcomes: a systematic review and meta-analysis. Heart 102(9):672-80

10. Schumacher K, Dagres N, Hindricks G, Husser D, Bollmann A, Kornej J (2017) Characteristics of PR interval as predictor for atrial fibrillation: association with biomarkers and outcomes. Clinical Res Cardiol [Epub ahead of print]. doi:10.1007/ s00392-017-1109-y

11. Kronborg MB, Nielsen JC, Mortensen PT (2010) Electrocardiographic patterns and long-term clinical outcome in cardiac resynchronization therapy. Europace 12:216-22

12. Bloch Thomsen PE, Jons C, Raatikainen MJ, Moerch Joergensen R, Hartikainen J, Virtanen V, Boland J, Anttonen O, Gang UJ, Hoest N, Boersma LV, Platou ES, Becker D, Messier MD, Huikuri HV (2010) Long-term recording of cardiac arrhythmias with an implantable cardiac monitor in patients with reduced ejection fraction after acute myocardial infarction: the Cardiac Arrhythmias and Risk Stratification After Acute Myocardial Infarction (CARISMA) study. Circulation 122(13):1258-1264

13. Ling LH, Kistler PM, Kalman JM, Schilling RJ, Hunter RJ (2016) Comorbidity of atrial fibrillation and heart failure. Nat Rev Cardiol 13(3):131-147

14. Moser JM, Willems S, Andresen D, Brachmann J, Eckardt L, Hoffmann E, Kuck KH, Lewalter T, Schumacher B, Spitzer SG, 
Hochadel M, Senges J, Hoffmann BA (2017) Complication rates of catheter ablation of atrial fibrillation in patients Aged $\geq 75$ years versus $<75$ years-results from the german ablation registry. $\mathbf{J}$ Cardiovasc Electrophysiol 28(3):258-265

15. Kutyifa V, Stockburger M, Daubert JP, Holmqvist F, Olshansky B, Schuger C, Klein H, Goldenberg I, Brenyo A, McNitt S, Merkely B, Zareba W, Moss AJ (2014) PR interval identifies clinical response in patients with non-left bundle branch block: a multicenter automatic defibrillator implantation trial-cardiac resynchronization therapy substudy. Circ Arrhythm Electrophysiol 7:645-651

16. Stockburger M, Moss AJ, Klein HU, Zareba W, Goldenberg I, Biton Y, McNitt S, Kutyifa V (2016) Sustained clinical benefit of cardiac resynchronization therapy in non-LBBB patients with prolonged PR-interval: MADIT-CRT long-term follow-up. Clinical Res Cardiol 105(11):944-952

17. Levey AS, Coresh J, Greene T, Stevens LA, Zhang YL, Hendriksen S, Kusek JW, Van Lente F (2006) Using standardized serum creatinine values in the modification of diet in renal disease study equation for estimating glomerular filtration rate. Ann Intern Med 145:247-254

18. Soliman EZ, Rautaharju PM (2012) Heart rate adjustment of PR interval in middle-aged and older adults. J Electrocardiol 45:66-69

19. Bazett HC (1920) An analysis of the time-relations of electrocardiograms. Heart 7:353-370

20. Pires LA, Abraham WT, Young JB, Johnson KM (2006) Clinical predictors and timing of New York Heart Association class improvement with cardiac resynchronization therapy in patients with advanced chronic heart failure: results from the Multicenter InSync Randomized Clinical Evaluation (MIRACLE) and Multicenter InSync ICD Randomized Clinical Evaluation (MIRACLEICD) trials. Am Heart J 151(4):837-843

21. Sanders P, Morton JB, Davidson NC, Spence SJ, Vohra JK, Sparks PB, Kalman JM (2003) Electrical remodeling of the atria in congestive heart failure: electrophysiological and electroanatomic mapping in humans. Circulation 108:1461-1468

22. Nikolaidou T, Cai XJ, Stephenson RS, Yanni J, Lowe T, Atkinson AJ, Jones CB, Sardar R, Corno AF, Dobrzynski H, Withers PJ,
Jarvis JC, Hart G, Boyett MR (2015) Congestive heart failure leads to prolongation of the PR interval and atrioventricular junction enlargement and ion channel remodelling in the rabbit. PLoS One 10:e 0141452

23. Aro AL, Anttonen O, Kerola T, Junttila MJ, Tikkanen JT, Rissanen HA, Reunanen A, Huikuri HV (2014) Prognostic significance of prolonged PR interval in the general population. Eur Heart J 35(2): 123-129

24. Crisel RK, Farzaneh-Far R, Na B, Whooley MA (2011) Firstdegree atrioventricular block is associated with heart failure and death in persons with stable coronary artery disease: data from the Heart and Soul Study. Eur Heart J 32(15):1875-1880

25. Januszkiewicz L, Vegh E, Borgquist R, Bose A, Sharma A, Orencole M, Mela T, Singh JP, Parks KA (2015) Prognostic implication of baseline PR interval in cardiac resynchronization therapy recipients. Heart Rhythm 12(11):2256-2262

26. Friedman DJ, Bao H, Spatz ES, Curtis JP, Daubert JP, AlKhatib SM (2016) Association between a prolonged PR interval and outcomes of cardiac resynchronization therapy: a report from the national cardiovascular data registry. Circulation 134(21):1617-1628

27. Whinnett $Z$ (2016) The His optimised pacing evaluated for heart failure trial (HOPE-HF). https://clinicaltrials.gov/ct2/show/ NCT02671903. Accessed Aug 2016

28. Holmqvist F, Thomas KL, Broderick S, Ersboll M, Singh D, Chiswell K, Shaw LK, Hegland DD, Velazquez EJ, Daubert JP (2015) Clinical outcome as a function of the PR-interval-there is virtue in moderation: data from the Duke Databank for cardiovascular disease. Europace 17:978-985

29. Nielsen JB, Pietersen A, Graff C, Lind B, Struijk JJ, Olesen MS, Haunso S, Gerds TA, Ellinor PT, Kober L, Svendsen JH, Holst AG (2013) Risk of atrial fibrillation as a function of the electrocardiographic PR interval: results from the Copenhagen ECG Study. Heart Rhythm 10:1249-1256

30. Cheng M, Lu X, Huang J, Zhang S, Gu D (2015) Electrocardiographic PR prolongation and atrial fibrillation risk: a metaanalysis of prospective cohort studies. J Cardiovasc Electrophysiol 26(1):36-41 